\title{
Cochlear microphonic enhancement in two tone interactions
}

\author{
Alfred L. Nuttall and David F. Dolan \\ The University of Michigan, Kresge Hearing Research Institute, Ann Arbor, Michigan, U.S.A.
}

(Received 14 April 1990; accepted 11 August 1990)

\begin{abstract}
Two tone interaction functions of the cochlear microphonic (CM) were obtained from pigmented guinea pigs. First (basal) cochlear turn recording locations show optimally enhanced levels of $\mathrm{CM}$ when the interfering tone $\left(\mathrm{F}_{2}\right)$ was positioned about $4 \mathrm{kHz}$ above probe tones $\left(F_{1}\right)$ of $12 \mathrm{kHz}$ and $20 \mathrm{kHz}$. Maximum enhancement occurred for equal level tones. No enhancement was seen for a probe tone of $4 \mathrm{kHz}$. When basal turn cochlear sensitivity was compromised, CM cnhancement causcd by the interfering tone was altered and only CM reduction was then seen. The CM reduction was the typical characteristic described by many earlier studies. Guinea pigs with various changes in cochlear sensitivity were studied, providing evidence in support of earlier reports that CM interference (both reductions and enhancements) depends on far field vector summation of the outputs of hair cells from a restricted area of the basilar membrane. No CM enhancement was seen in micropipette recordings from within the organ of Corti.
\end{abstract}

Guinea pig; Two tone suppression; Active process; Physiological vulnerability; Phase effects; Vector cancellation

\section{Introduction}

When two pure tones simultaneously stimulate the cochlea a variety of nonlinear phenomena are revealed in the cochlear potentials. These phenomena have been vigorously studied for more than fifty years. Even so, details of cochlear electrophysiology continue to emerge and provide a basis for complete understanding of function and pathology. This report characterizes a type of nonlinear behavior in the cochlear microphonic (CM) that largely appears to have escaped previous observation although it is a prominent feature of two tone interactions in the guinea pig. The finding is a form of CM interference but it is an enhancement rather than a decrease in the magnitude of the probe tone caused by the interfering tone.

C.M interference was first noted by Covell and Black (1936) and soon was the subject of specific research studies (Bray and Thurlow, 1942; Wever et al., 1940; Wever and Lawrence, 1941). Nieder

Correspondence to: Alfred L. Nuttall, The University of Michigan, Kresge Hearing Research Institute, 1301 E. Ann Street, Ann Arbor, MI 48109-0506, U.S.A. and Nieder $(1968 \mathrm{a}, \mathrm{b})$ studied the phenomenon for the information conveyed about cochlear nonlinearity while Legouix and colleagues (Legouix et al., 1973; Legouix et al., 1976; Legouix and Remond, 1977) proposed that interference was analogous to neural and psychophysical two-tone suppression. An important difference between neural two tone suppression and CM interference is that suppression is strongest ncar (above and bclow) the probe frequency, while $\mathrm{CM}$ interference is strongest when the interfering-tone frequency is higher than the probe. Interference is a small effect when the two tones are of nearly equal frequency or when the interference-tone is lower in frequency than the probe. This difference was noted by Dallos et al., (1974) and more thoroughly explored by Cheatham and Dallos (1982). The latter authors proposed that $\mathrm{CM}$ interference is partially the result of phase cancellation in $\mathrm{CM}$ recordings.

As the term interference suggests, the change occurring in $\mathrm{CM}$ that was noted in previous studies is a reduction in CM magnitude. Toward understanding the mechanism behind interference, Cheatham and Dallos (1982) proposed a phenomenological model based on vector summation, at 
the recording electrode, of $\mathrm{CM}$ from remotely located generators (hair cells). In their model, CM interference is the result of changes in the outputs of some of the $\mathrm{CM}$ generators which contribute to the vector sum. Since hair cells arranged along the basilar membrane have differing response phases (due to travelling wave propagation time), reduction in the magnitude or alteration of the phase of some hair cells will cause a change in the ensemble vector sum. The factor which causes changes in some of the CM generators is presumably two-tone suppression occurring in the region of the organ of Corti where the two-tone travelling waves are jointly active. Removal of energy from some generators (by two tone suppression) can potentially result in any combination of change in the ensemble resultant magnitude and phase.

Thus the mechanism of interference includes those phenomena that cause two tone suppression. The term 'two tone suppression' originally emerged in the literature to describe observations on neural responses. However, this historical usage of the term has been extended to basilar membrane mechanics and inner hair cell receptor potentials which almost certainly are the basis for the neural effects. The term 'two tone interference' predates suppression but it has generally been used to describe the $\mathrm{CM}$ changes.

In the current study we show strong (up to $50 \%$ ) increases (as well as decreases) in first cochlear turn recordings for frequencies above approximately $8 \mathrm{kHz}$ in the guinea pig. The increases are physiologically vulnerable. In the cochlea exhibiting less than normal sensitivity, the $\mathrm{CM}$ changes only exhibit the well known frequency specific decrease above the probe frequency. On the other hand, extracellular ac receptor potentials, recorded within the organ of Corti in normal cochleas, only show suppression (i.e. never an increased receptor potential). This observed suppression of CM, within the organ of Corti is most likely related to two tone intermodulation in responses of outer hair cells. It has been proposed that mechanical basilar membrane suppression has linked these changes in outer hair cell receptor current (Geisler et al., 1990; Patuzzi et al., 1989a). We conclude that the data on CM enhancement is also consistent with a vectoral summation hypothesis and that enhancement is dependent on normal outer hair cell function (i.e. the array of magnitudes and phases present in the normal cochlea).

\section{Methods}

Pigmented guinea pigs (with body weights 250 $350 \mathrm{~g}$ ) were pre-anesthetized with nembutal (15 $\mathrm{mp} / \mathrm{Kg} \mathrm{IP}$ ) and surgical ancsthesia was achicved after administration of Innovar-Vet $(0.4 \mathrm{mg} / \mathrm{Kg}$ IM). Premedication with Atropine $(0.05 \mathrm{mg} / \mathrm{Kg}$ SC) was used to reduce respiratory secretions. Supplemental doses of Innovar-Vet and Nembutal (at $1 / 2$ the initial amount) were given every 1 and $3 \mathrm{~h}$, respectively. The animals were wrapped in a heating blanket, which maintained rectally measured temperature at $38 \pm 1^{\circ} \mathrm{C}$. Heart rate was monitored. A tracheal intubation was performed to maintain an airway, but the animals were not artificially respirated. A ventral surgical dissection of the neck exposed the auditory bulla which was opened widely without compromising the ossicles or tympanic membrane. The head had previously been placed into a head holder, held by clamps over the zygomatic arches. Heat was supplied to the head by a head holder heater and from a lamp directed onto the surgical area. This prevented cooling of the cochlea and reduction of high frequency sensitivity (Brown et al., 1983; Shore and Nuttall, 1985). The tensor tympani muscle tendon was severed to prevent its spontaneous contractions from interfering with electrical measurements from the cochlea.

Sounds were delivered to the external auditory meatus by a closed system. The speculum that was fitted into the meatus contained a $1 / 2$ inch Bruel and Kjear ( $\mathrm{B}$ and $\mathrm{K}$ ) condenser microphone which acted as a sound source and a $1 \mathrm{~mm}$ probe tube/microphone system for calibration of the sound levels. The probe microphone, which itself was calibrated against a $1 / 8$ inch $B$ and $K$ condenser microphone in a small coupler, was positioned to within $2 \mathrm{~mm}$ of the tympanic membrane for sound calibration measurements. Sounds presented to the animal were given in the form of 150 $\mathrm{ms}$ tone bursts ( $10 \mathrm{~ms}$ rise/fall time) at the rate of five tones per second from computer controlled attenuators and tone switches (Wilsonics PATT and BSIT). Three different frequencies of probe 
tone $\left(F_{1}\right)$ were used $(4,12$, and $20 \mathrm{kHz})$ at 5 levels $(40,50,60,70$, and $80 \mathrm{~dB}$ SPL). For every combination of $F_{1}$ (i.e. each frequency and intensity), a tonal complex of $F_{1}$ and an interfering tone $F_{2}$ was also given. The interfering tone frequency was varied from 2 to $28 \mathrm{kHz}$ in $1 \mathrm{kHz}$ steps and in level from 35 to $85 \mathrm{~dB}$ SPL. There were 8 levels of $\mathrm{F}_{2}(35,40,50,60,70,80$, and $85 \mathrm{~dB}$ SPL $)$. A PC-AT type microcomputer controlled the sounds and stored the magnitude and phase of the resultant cochlear microphonic as measured by a lock-in amplifier (Stanford Model SR530). The reference frequency of the lock-in amplifier was always the electrical signal taken directly from the $F_{1}$ oscillator. An interference value was calculated by dividing the $C M$ value for $F_{1}$ and $F_{2}$ given together by the $C M$ value of $F_{1}$ alone. Likewise, phase change was calculated as the phase of the tonal combination minus the value of the phase for $F_{1}$ alone. Therefore, a phase advance (lead) is a positive value.

A total of 51 guinea pigs were used in this study. Most had measurements done from a single wire electrode on the round window membrane (40 gauge Teflon insulated silver wire) with the reference electrode in the neck muscles (a chlorided 26 gauge silver wire). In some experiments ( 9 guinea pigs) differential electrodes were fixed into the first (basal) cochlear turn. In this case two wires (40 gauge Teflon insulated silver wire) were placed into holes drilled into the bony cochlear wall, one into scala tympani and the other into scala vestibuli. Differential measurements were always referred to a common ground in the neck muscles. The scala tympani electrode went to the non-inverting input of the Grass P16 preamplifier.

In some experiments (10 guinea pigs), an opening in the scala tympani of the first turn of approximately $0.5 \mathrm{~mm}$ diameter was made in a manner similar to the technique used to prepare a guinea pig for intracellular recording from hair cells (Brown and Nuttall, 1984). The basilar membrane was visualized using a stereo-microscope while a glass micropipette electrode was advanced into the organ of Corti. The electrode was filled with $5 \mathrm{M}$ potassium acetate and had a tip size of 1 $\mu \mathrm{m}$. This is a relatively low impedance electrode which improved the signal levels available at high frequencies. Optimal amplifier negative capaci- tance compensation was used for recordings from the glass electrode. Recordings from the organ of Corti were made from a location in the tunnel of Corti.

The normality of cochlear sensitivity was assessed by recording the threshold CAP from the round window electrode. This was done before and after all tones of a series were given. The sensitivity was also measured before and following surgical procedures such as placement of holes for the differential electrodes. This study used a large number of animals in order to measure interference at a variety of cochlear performance levels, both normal and abnormal, the most interesting of which are described below. CM interference functions are quite consistent across animals and thus only representative examples are used in the Results.

\section{Results}

The phenomenon of $\mathrm{CM}$ interference is illustrated in Fig. 1. The data were obtained by combining a probe tone of fixed frequency (12 or $20 \mathrm{kHz}$ ) at a constant sound level ( $\approx 60 \mathrm{~dB}$ SPL) with a second tone (the interfering sound) also having a constant sound level $(\approx 60 \mathrm{~dB}$ SPL) but varying in frequency between 2 and $28 \mathrm{kHz}$. In panels $\mathrm{A}$ and $\mathrm{C}$ of the figure the ordinate gives the normalized CM produced by the probe tone combined with the interfering tone. Panels $B$ and $D$ show the change in the phase angle of the probe tone caused by the interfering tone. The results given in Fig. 1 were unexpected because a prominent effect of the interfering tone was to cause a substantial frequency dependent increase in the level of $\mathrm{CM}$ produced by the probe. Previous studies had detailed only decreased CM in this frequency region above the probe frequency (eg. Legouix et al., 1976; Cheatham and Dallos, 1982).

The tonal conditions leading to the maximum $\mathrm{CM}$ are quite characteristic in these round window recordings. The interfering tone must be approximately $4 \mathrm{kHz}$ higher than the frequency of the probe tone and their levels approximately equal. The examples given in Fig. 1 for frequencies of $12 \mathrm{kHz}$ and $20 \mathrm{kHz}$ (panels $\mathrm{A}$ and $\mathrm{C}$, respectively) show the enhancement maxima at 16 and $24 \mathrm{kHz}$ respectively. If the interfering tone 
sound level is higher than the probe, the enhancement is lessened and eventually causes reduced $\mathrm{CM}$ levels (for sufficiently loud interfering tones). If a probe tone is below approximately $\approx 8 \mathrm{kHz}$ the enhancement phenomenon is lessened and the phenomenon is not observed with probe tones of 4 $\mathrm{kHz}$ or below.

The interaction of the interfering tone with the probe is shown more completely in Fig. 2. These graphs were constructed by a surface fitting algorithm (a generalized parabolic interpolation) to data points of change of the probe for 22 frequencies of $F_{2}$ and 7 sound levels of $F_{2}$. Like Fig. 1, panels $\mathrm{A}$ and $\mathrm{C}$ are the magnitude change while $\mathrm{B}$ and $D$ are the phase changes. Since this figure gives the data from the same animal as Fig. 1, each line graph of Fig. 1 is simply a cut through the surfaces at the $60 \mathrm{~dB}$ SPL $F_{2}$ sound level. The similarity of the interference effect at the two probe frequencies is evident. Enhancement of the probe characteristically occurs at equal sound levels and for an interfering frequency above that of the probe tone. The phase goes through a lag/lead inflection.

The apparent active area of the organ of Corti seen in far field signal recordings can be significantly restricted with the use of differential electrode arrangements (Dallos, 1973). Fig. 3 shows that the enhancement/decrement character of the interference function is preserved when recorded differentially. In Fig. 3A, the surface type plot shows a much more sharply defined pattern of interference than Fig. 2A but the general form is the same (note that the direction of the $F_{2}$ frequency range is reversed in order to allow visualization of the reduction). A cut through the
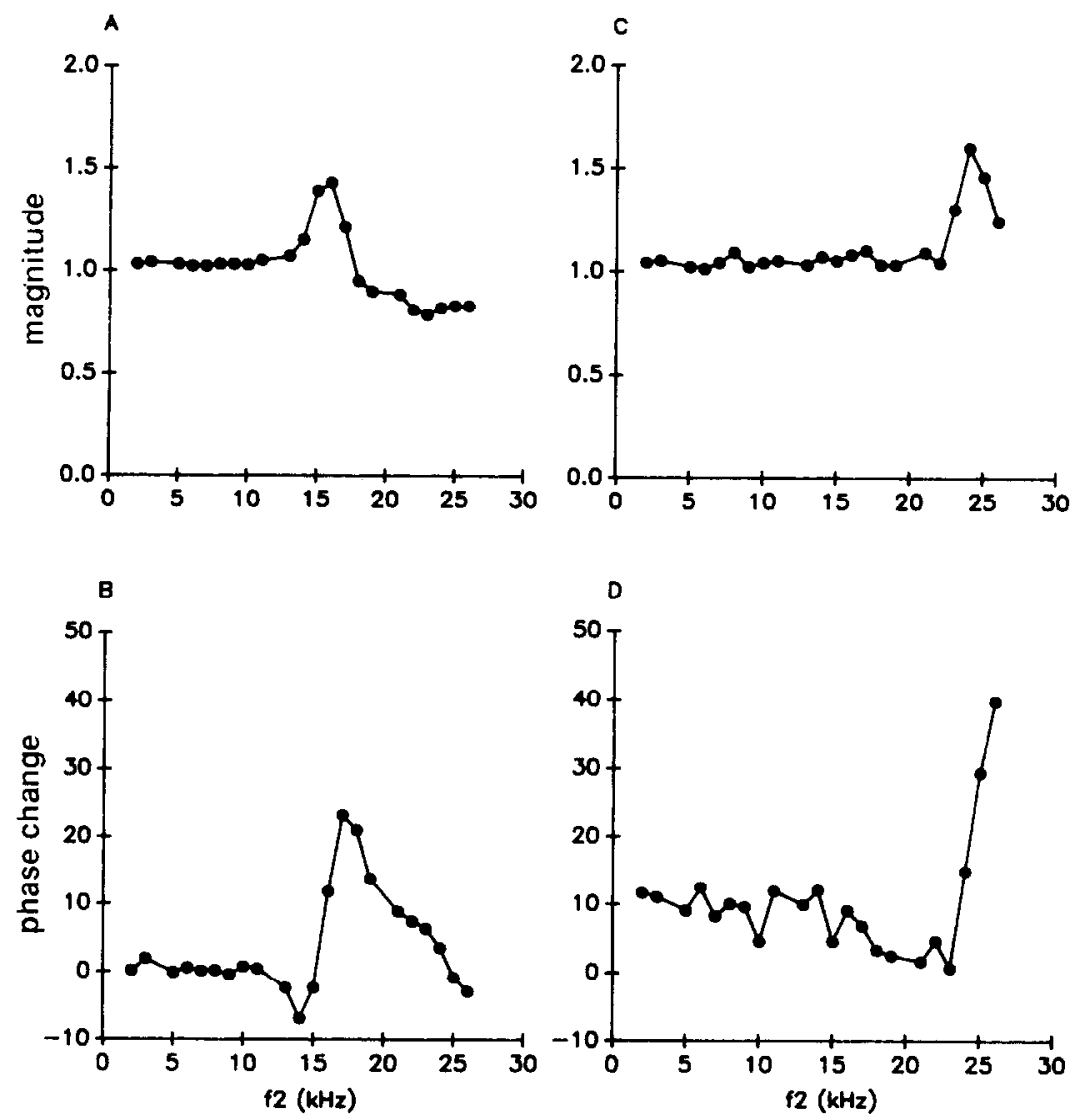

Fig. 1. The influence of an interfering tone $\left(F_{2}\right)$ on the magnitude and phase of a probe tone $\left(F_{1}\right)$ [recorded from the round window membrane]. In panels $\mathrm{A}$ and $\mathrm{B}$, the probe tone frequency was $12 \mathrm{kHz}$. In panels $\mathrm{C}$ and $\mathrm{D}$ the probe tone frequency was $20 \mathrm{kHz}$. The magnitude level of the probe and interfering tone was $60 \mathrm{~dB}$ SPL. 
surface at the $60 \mathrm{~dB}$ level shows the more restricted frequency range of $F_{2}$ influence (Fig. 3C). The phase function, like the round window data, goes through a phase reversal but it is much more pronounced. Maximum enhancement in the differentially measured $\mathrm{CM}$ occurred at $14 \mathrm{kHz}$. The point of phase transition, however, appears to be at the same frequency in both measurement schemes $(16 \mathrm{kHz})$.

$\mathrm{CM}$ enhancement is a function of the physiological condition of the cochlea. In the guinea pig basal turn, high frequency sensitivity is readily lost during surgical procedures and in other conditions such as decreased cochlear temperature (Brown et al., 1983; Shore and Nuttall, 1985). The loss of normal basal turn sensitivity is associated with decreased inner hair cell tuning and elevated CAP thresholds. Fig. 4 shows that the loss of normal cochlear performance for the basal turn results in conversion of the magnitude interference function recorded by differential electrodes to one showing only $\mathrm{CM}$ reduction. Likewise the phase shift is converted from the pronounced lead/lag condition of Fig. 3D to largely a lagging phase function (Fig. 4B). A second example of the loss of CM enhancement is given is Fig. 5. In this case however the guinea pig expressed an unusual form of cochlear pathological performance. There was pronounced loss of cochlear sensitivity only for frequencies below $20 \mathrm{kHz}$ as shown by the CAP audiogram in Fig. 6. Note, in Fig. 5, that the magnitude and phase functions for the $12 \mathrm{kHz}$ probe show the same elimination of enhancement of $\mathrm{CM}$ and phase shift inflection as does the differentially recorded result in a different animal (Fig. 4). On the other hand, the functions recorded from the 'normal' area of the organ of Corti (Fig. $5 \mathrm{C}$ and $\mathrm{D}$ ) appear quite similar to those for the $20 \mathrm{kHz}$ probe given in Fig. 1. The topographic characteristic frequency locations of 12 and 20 $\mathrm{kHz}$ are separated by about $1.8 \mathrm{~mm}$ (Robertson and Johnstone, 1979) in the guinea pig cochlea suggesting that the interference phenomenon leading to increased $\mathrm{CM}$ is derived from a narrow region of the basilar membrane.

When a $\mathrm{CM}$ enhancement is seen at the round window or in differential recordings one may justifiably inquire: Are the receptor potentials of
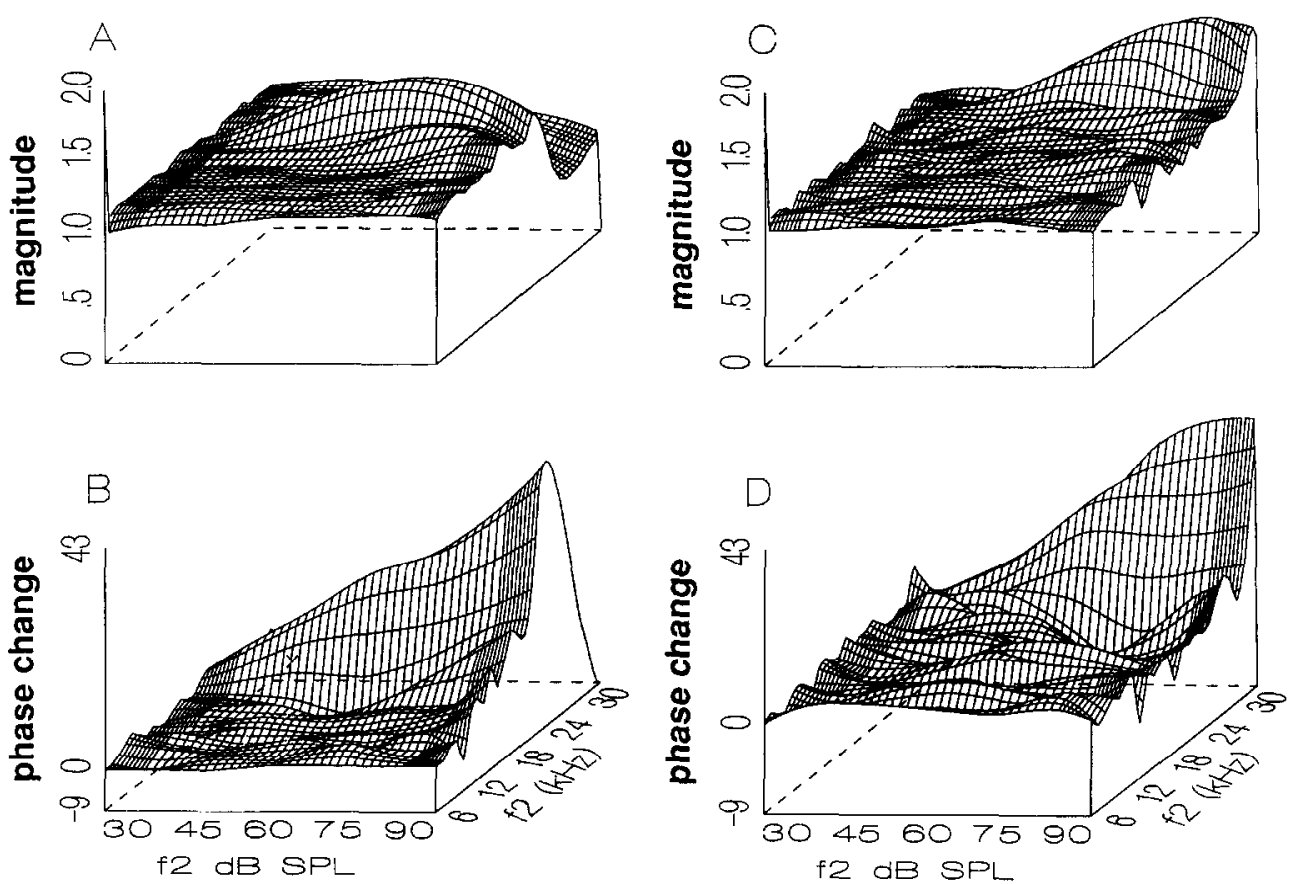

Fig. 2. The influence of an interfering tone $\left(F_{2}\right)$ on the magnitude and phase of a probe tone recorded from the round window membrane. Panels A and B are for a probe tone of $12 \mathrm{kHz}$. Panels $\mathrm{C}$ and D are for a probe tone of $20 \mathrm{kHz}$. These surface plots show the influence of interfering tone magnitude as well as frequency on the level and phase of the probe tone. 
outer hair cells actually increased because of the effects of $F_{2}$ ? This question can be examined by measuring the extracellular potentials in the organ of Corti using a microelectrode. With the microelectrode technique the recorded signal represents a measurement of generators localized very close to the electrode tip (eg. Patuzzi et al., 1989b). Fig. 7 shows the interference function obtained in a normal cochlea from an electrode located in the tunnel of Corti at about the $20 \mathrm{kHz}$ best frequency location along the basilar membrane. The surface has an irregular appearance because of the low signal to noise level when recording high frequencies from a microelectrode. However it is clear that the function only exhibits a reduction in the microelectrode recorded potential. This occurs in a characteristic fashion that essentially follows the shape of the tuning curve of the basilar membrane location. At lower frequencies and at higher frequencies little reduction of the recorded signal is

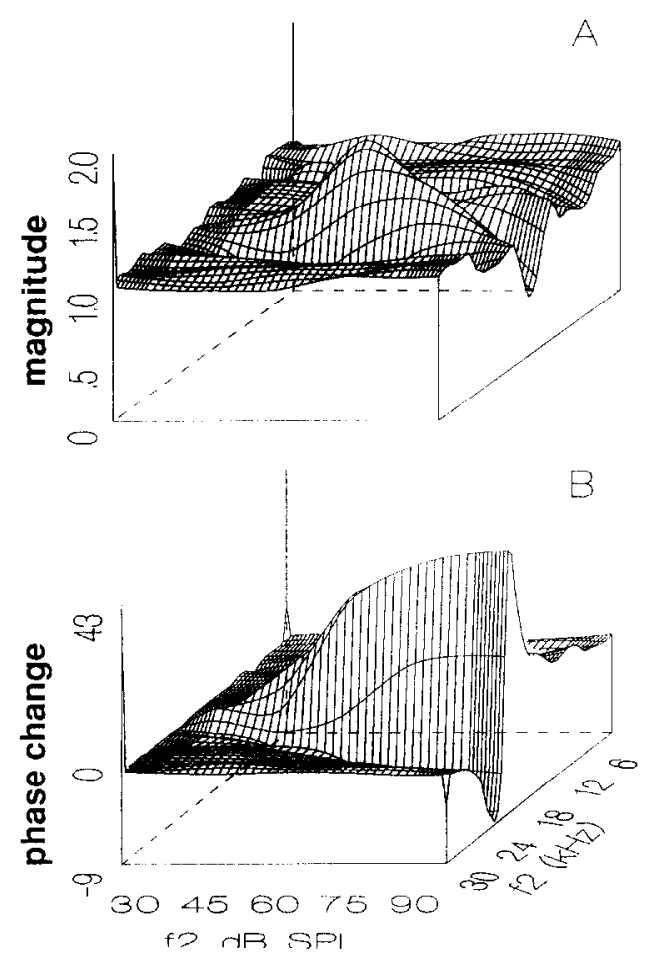

evident. This intra-organ of Corti measurement is consistent with the concept of two tone suppression.

\section{Discussion}

The purpose of this report is to describe the phenomenon of $\mathrm{CM}$ enhancement in the guinea pig cochlcar microphonic. It is closely linked with and is indeed dependent upon the highly vulnerable sensitivity and frequency selectivity of the first (basal) turn. The phenomenon is a normal and prominent feature of two tone interactions but to our knowledge, has not been previously reported.

CM interference has had a long history of investigative study beginning with those of Black and Covell (1936). It remained a somewhat puzzling two tone interaction eventually giving rise to the hypothesis that it was the cochlear potential

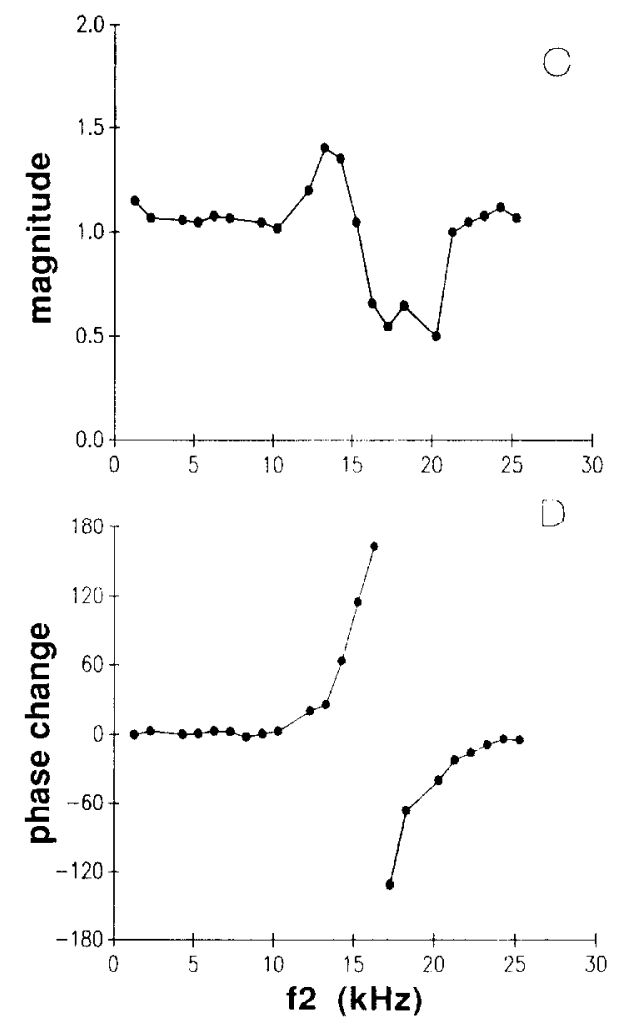

Fig. 3. Differential (first turn) electrode recordings of the interference effect. Panels $A$ and $B$ give the magnitude and phase of the interference effect on a probe tone of $12 \mathrm{kHz}$. The surface plots show a more sharply defined magnitude enhancement followed by a region of CM reduction, the 'dip' frequency area. Panels $C$ and D represent a cut through the surface plot at the $60 \mathrm{~dB}$ level for $F_{2}$. 


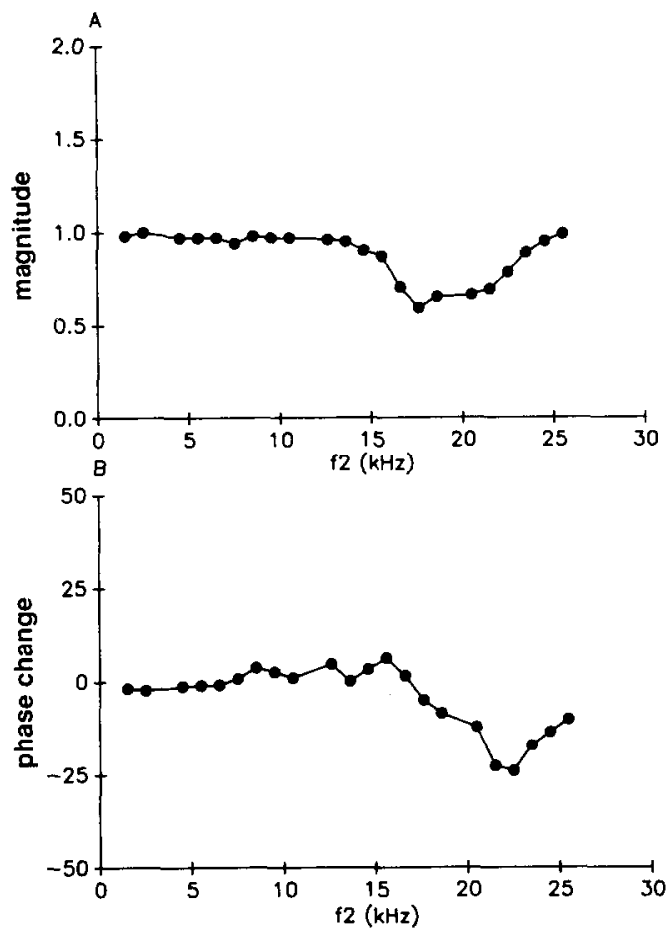

Fig. 4. The magnitude and phase of the interference effect of a probe tone of $12 \mathrm{kHz}$ in a first turn differential recording from an animal which had abnormal cochlear sensitivity. There is a loss of the enhancement effect in the magnitude function.

manifestation of two tone suppression (Legouix et al., 1976). However Dallos et al. (1974) suggested that the $\mathrm{CM}$ reduction caused by the interfering tone was due to cochlear electrical effects combined with suppression. Cheatham and Dallos (1982) published a comprehensive parametric study of CM interference and proposed a phenomenological model to account for it based on interactions in the gross cochlear potentials. The results of the current study are consistent with the general concept of their model.

No previous studies report $\mathrm{CM}$ enhancement in the frequency region significantly above the probe. Cheatham and Dallos (1982) show an increase in $\mathrm{CM}$ level for a frequency range of $F_{2}$ positioned at or slightly below $F_{1}$, and Legouix et al (1976) say that additive effects can occur when $F_{2}$ is close to $F_{1}$. All previous reported interactions of $F_{2}$ above $F_{1}$ describe a distinct 'dip' frequency for $F_{2}$. We propose that this is partially due to the choice of probe tone frequencies well below $12 \mathrm{kHz}$ in most previous studies, but also that changes in basal turn cochlear sensitivity can be implicated. Loss of normal function would obscure the observation of the enhancement effect.

$\mathrm{CM}$ enhancement would be difficult to account for if one resorted only to two tone suppression as a rationale. Enhancement is perhaps the strongest evidence for rejecting the idea that this tonal interaction mimics the shape of psychophysical or neural suppression functions. CM enhancement and decrements can readily be accounted for by a vector summation model such as that proposed by Cheatham and Dallos (1982). This model treats the resultant electric potential recorded by an electrode on the round window, or electrodes positioned as a differential pair across the organ of Corti, as the sum of many elemental generators (the hair cells) arranged as spatial array and having the phase shifts imposed by the underlying displacement traveling wave of the basilar membrane. The explanation of CM interference then includes both two tone suppression and vector summation. An $\mathrm{F}_{2}$ tone will cause a certain degree of two-tone suppression for a specific spatial group of generators in the interaction area of the basilar membrane where the traveling waves of the two tones are common. The electric potential seen by a far field recording system, for the outer hair cell responding to the travelling wave due to $F_{1}$, will be changed by the second tone which removes (by two tone suppression) the vector contributions of some hair cells. Depending on the specific mix of vectors the resultant could experience phase advance or lag and the magnitude could increase or decrease.

The process of two tone enhancement does not have a counterpart in the performance of auditory afferent fibers or inner hair cell receptor potentials. Likewise, in the current study, an extracellular electrode recording in the tunnel of Corti (which receives locally produced outer hair cell receptor potentials) revealed only reduced $\mathrm{CM}$ (Fig. 7). Thus, even though a far field electrode records enhanced $\mathrm{CM}$, outer hair cells themselves simultaneously have either suppressed or unchanged receptor potentials. Geisler et al. (1990) have provided evidence that the local reduction 

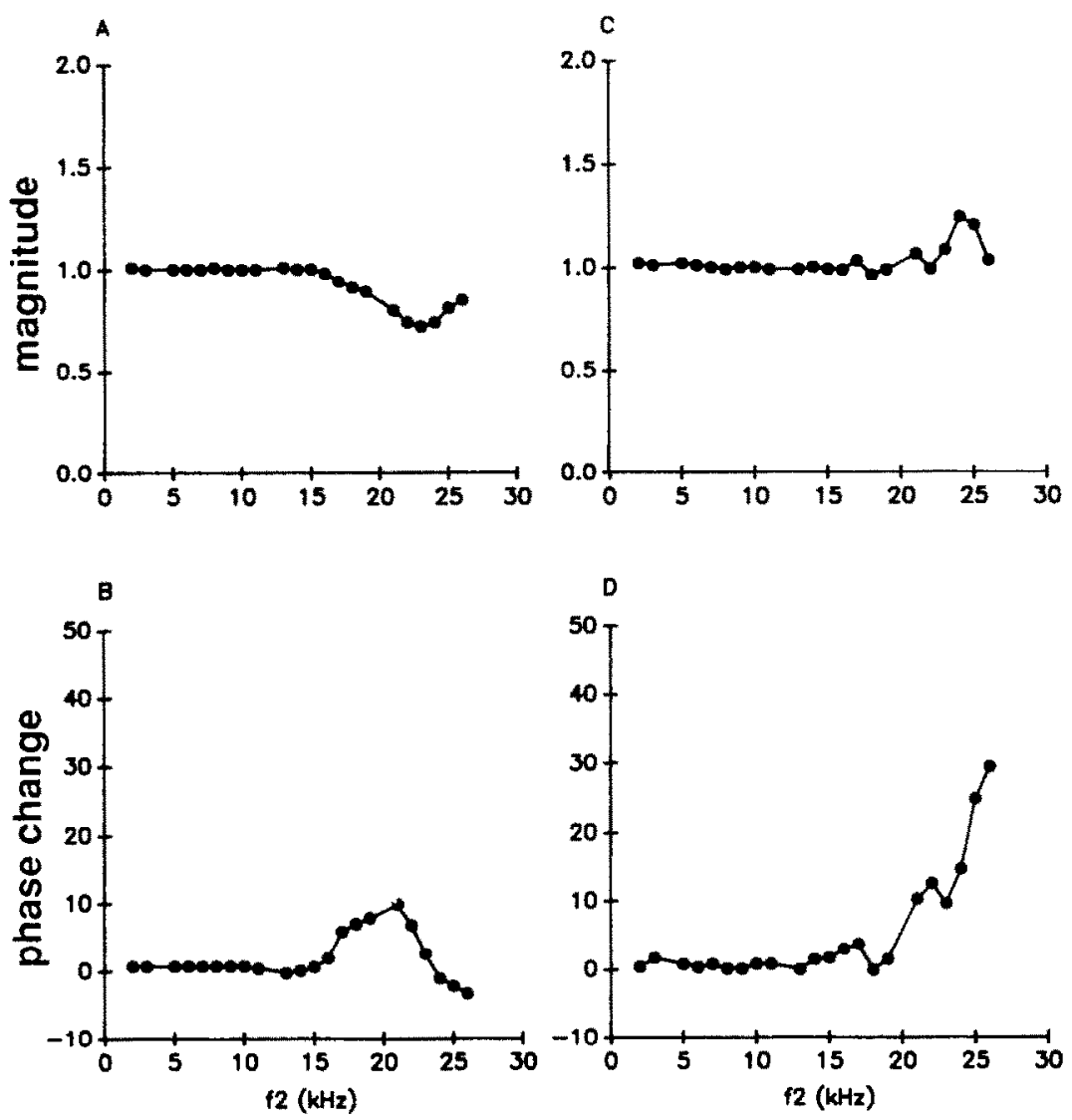

Fig. 5. Magnitude and phase interference functions from a round window electrode showing loss of $\mathrm{CM}$ enhancement for a $12 \mathrm{kHz}$ probe in panels $A$ and $B$, and preservation of $C M$ enhancement in panels $C$ and $D(20 \mathrm{kHz})$. The cochlear sensitivity in this animal had abnormal hearing below $20 \mathrm{kHz}$ (see Fig. 6).

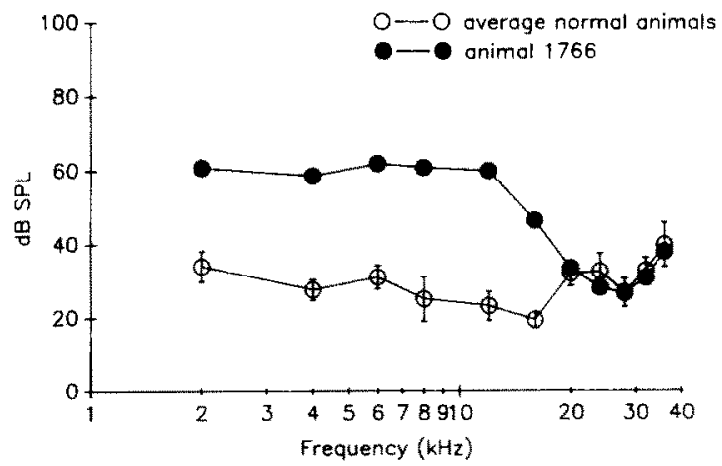

Fig. 6. Compound action potential audiogram from the round window of an animal having an unusual low-frequency sensitivity loss with preservation of normal sensitivity above $20 \mathrm{kHz}$ (closed circles). This was the animal whose magnitude and phase interference functions are depicted in Fig. 5. Mean threshold and standard deviation bars are given for 10 normal cochleas (open circles). (i.e. within the organ of Corti) in ac responses represents a shift of energy into intermodulation distortion as the outer hair cells are driven into saturation by one or both of the two tones. The sound levels which just begin to produce outer hair cell receptor current saturation also cause suppression of inner hair cell dc receptor potentials. If the reduced extracellular potentials (which are proportional to outer hair cell receptor current) are directly correlated with an active mechanical drive to the basilar membrane as proposed by Patuzzi et al. (1989a), then one may think of two tone $\mathrm{CM}$ interference as a complicated measure of the cause of two tone suppression (i.e. reduction in outer hair cell receptor current). Cer- 


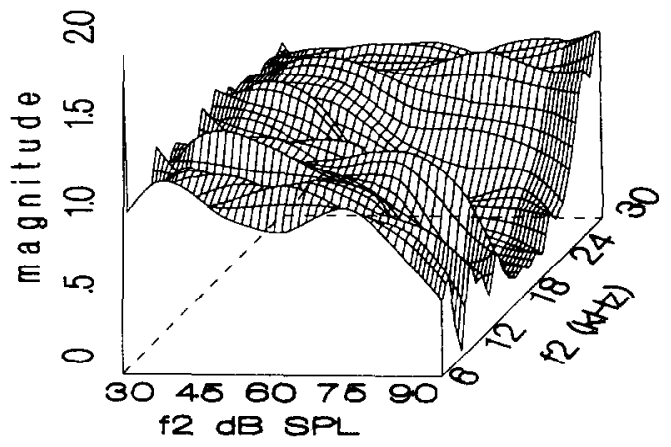

Fig. 7. A surface plot of the magnitude of change of the cochlear microphonic for a $12 \mathrm{kHz}$ probe tone recorded by a micropipette electrode placed in the tunnel of Corti at the 18 $\mathrm{kHz}$ CF location. No significant enhancement is seen when $\mathrm{CM}$ is recorded from within the organ of Corti.

tainly Legouix et al. (1976) and Dallos et al. (1974) were quite correct in relating CM interference to two tone suppression.
What is it then about the normal sensitivity of the basal turn which produces the $\mathrm{CM}$ enhancement? We offer the following qualitative rationale invoking an extension of the vector summation idea. Recall that in the normal cochlea travelling waves for tones with basal turn characteristic frequencies (CFs) are strongly peaked displacement functions (e.g. Khanna and Leonard, 1982; Sellick et al., 1982). A round window electrode may then be thought to receive contributions from proximate generators (Patuzzi et al., 1989) but those from near the traveling wave peak will dominate. The phase shifts among these vectors will be large and most of the signal will come from the basal aspect (lower sloped side) of the traveling wave. Thus the interfering tone will tend to have its most complex effects when it is of higher frequency. The $F_{2}$ travelling wave will suppress out of phase and
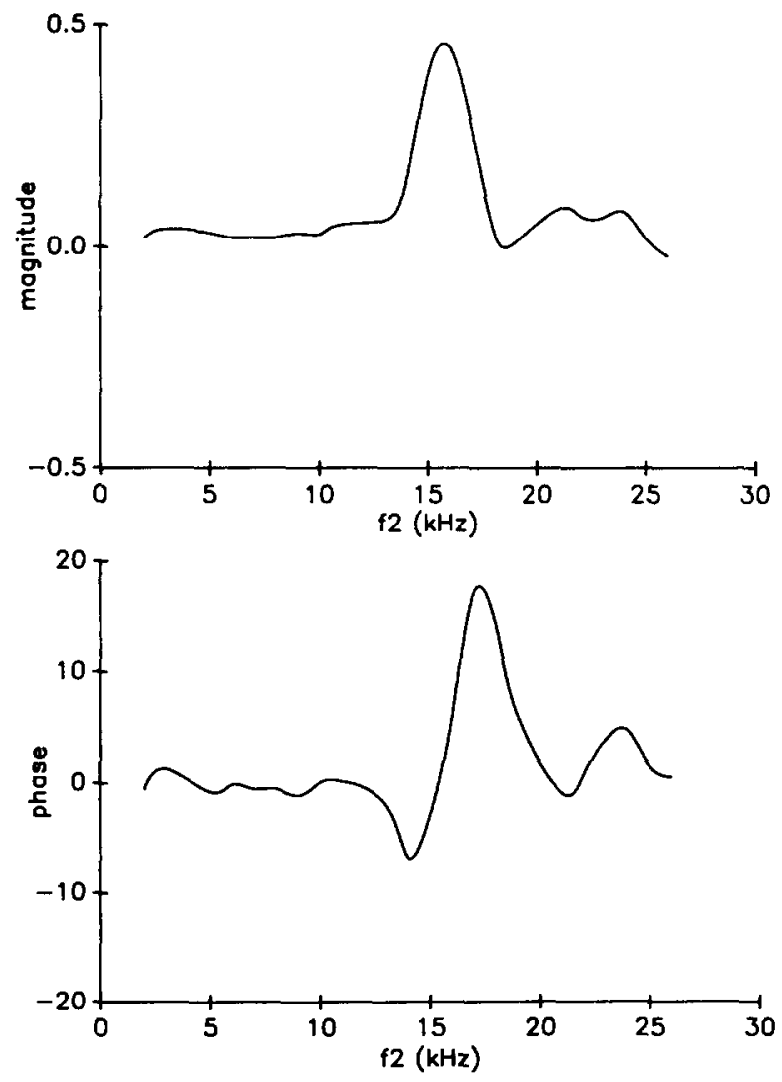

Fig. 8. Difference functions showing the residual magnitude and phase obtained by subtracting the interference functions of the abnormally sensitive cochlea from the normal cochlea. Probe tone was $12 \mathrm{kHz}$ at $60 \mathrm{~dB}$ SPL. Interfering tone was $60 \mathrm{~dB}$ SPL. 
lower magnitude-level vectors. The resultant vector would be increased only if significant vector power is in phase opposition in the normally sensitive cochlea.

In Fig. 5, data from a guinea pig with near normal high frequency sensitivity at $20 \mathrm{kHz}$ and poor sensitivity at $12 \mathrm{kHz}$ show that the enhancement was absent when $F_{1}$ was positioned at 12 $\mathrm{kHz}$. Thus the $\mathrm{CM}$ enhancement property for a 12 $\mathrm{kHz}$ probe tone (in a normally performing cochlea) would likely come from hair cells positioned between the 12 and $20 \mathrm{kHz} \mathrm{CF}$ locations. It is interesting in this regard to examine the difference function derived from the normal and abnormal interference functions. Fig. 8 shows such a function constructed by subtracting interpolated values of Figure $1 \mathrm{~A}$ and $\mathrm{B}$ from Fig. $5 \mathrm{~A}$ and $\mathrm{B}$ and fitting the points with a smooth curve. Note that the magnitude enhancement is accompanied by a lead/lag phase shift suggesting that the interfering tone covers a large range of phase delay in the probe. One could interpret the difference function as indicating extra phase shift added by the normally functioning outer hair cells, but a more conservative view would be that the active biomechanics of the organ of Corti simply enhance the magnitude of a range of phase delay already present on the basilar membrane. Whichever of these viewpoints is correct, it is clear from the current data that the frequency of best enhancement is a constant difference between the primaries of about $4 \mathrm{kHz}$. A similar observation was made by Dallos et al. (1974) that the 'dip' frequency shifted directly with frequency changes in the probe. Since the phenomenon of CM enhancement seems to be related to a narrow range of hair cells along the basilar membrane, its occurrence is more strongly associated with the actual magnitudes and phases of the hair cell receptor potentials than with the complex electroanatomy of the far field recording situation.

\section{Acknowledgments}

The authors thank Dr. M. Ruggero for reviewing the manuscript and C.M. Hall for help with data analysis and preparation of figures in this report. The study was supported by funding from NIH NS15107 and NS05785.

\section{References}

Black, L.J. and Covell, W.P. (1936) A quantitative study of the cochlear response. Proc. Soc. Exp. Bio. Med. 33, 509-511.

Bray, C.W. and Thurlow W.R. (1942) Interference and distortion in the cochlear responses of the pigeon. J. Comp. Psychol. 33, 279-289.

Brown, M.C. and Nuttall, A.L. (1984) Efferent control of cochlear inner hair cell responses in the guinea-pig. $J$. Physiol. 354, 625-656.

Brown, M.C., Smith, D.I. and Nuttall, A.L. (1983) The temperature dependency of neural and hair cell responses evoked by high frequencies. J. Acous. Soc. Am. 73, 1662-1670.

Cheatham, M.A. and Dallos, P. (1982) Two-tone interactions in the cochlear microphonic. Hear. Res. 8, 29-48.

Covell, W.P. and Black, L.J. (1936) The cochlear response as an index to hearing. Am. 1. Physiol. 116, 524-530.

Dallos, P., Cheatham, M.A. and Ferraro, J. (1974) Cochlear mechanics, nonlinearities and cochlear potentials. J. Acoust. Soc. Am. 55, 597-605.

Dallos, P. (1973) The Auditory Periphery: Biophysics and Physiology, Academic Press. NY.

Geisler, C.D., Yates, G.K. and Patuzzi, R.B. (1990) Saturation of outer hair cell receptor currents causes two tone-suppression. Hear. Res. 44, 241-256.

Khanna, S.M. and Leonard, D.G.B. (1982) Basilar membrane tuning in the cat cochlea. Science 215, 305-306.

Legouix, J.P., Remond, M.C. and Greenbaum, H.B. (1973) Interference and two tone inhibition. J. Acoust. Soc. Am. $53,409-419$.

Legouix, J.P., Remond, M.C. and Harrison, J. (1976) Interference, its functional significance. In: S.K. Hirsch et al. (Eds.), Hearing and Davis: Essays honoring Hallowell Davis, Washington University Press, St. Louis, MO, pp. 53-63.

Legouix, J.P. and Remond, M.C. (1977) The phenomenon of interference. Variations with physical and physiological factors. In: XIV Workshop Inner Ear Biology, Bordeaux, INSERM, Paris. pp. 93-102.

Nieder, P. and Nieder, 1. (1968a) Some effects of tonal interactions as seen in the cochlear microphonic. J. Acoust. Soc. Am. 43, 1092-1106.

Nieder, P. and Nieder, L. (1968b) Sorne studies of low-tone interaction as seen in the guinea pig microphonic. J. Acoust. Soc. Am. 44, 1409-1422.

Patuzzi, R.B., Yates, G.K. and Johnstone, B.M. (1989a) Outer hair cell receptor current and sensorineural hearing loss. Hear. Res. 42, 47-72.

Patuzzi, R.B., Yates, G.K. and Johnstone, B.M. (1989b) The origin of the low-frequency microphonic in the first cochlear turn of the guinea pig. Hear. Res. 39, 177-188.

Robertson, D. and Johnstone, B.M. (1979) Aberrant tonotopic 
organization in the inner ear damaged by kanamycin. $J$. Acoust. Soc. Am. 66, 466-469.

Shore, S.E. and Nuttall, A.L. (1985) The effects of cochlear hypothermia on compound action potential tuning. $J$. Acoust. Soc. Am. 77, 590-598.
Wever, E.G., Bray, C.W. and Lawrence, M. (1940) The interference of tones in the cochlea. J. Acoust. Soc. Am. 12, 268-280.

Wever, E.G. and Lawrence, M. (1941) Tonal interference in relation to cochlear injury. J. Exp. Psychol. 29, 283-295. 